\title{
A new LMS algorithm and its application to improve quality of broadcast-telephone system use in underground coal mines
}

\author{
Truyen N.T $T^{a}$ and Vinh N.T \\ ${ }^{a}$ Vietnam Research Institute of Electronics, \\ Informatics and Automation, Vietnam \\ ${ }^{\mathrm{b}}$ Electronics and Automation Technology \\ Development Company, Vietnam \\ E-mail:dthv@yahoo.com,ntvcie@yahoo.com
}

\begin{abstract}
-
A new LMS algorithm with a variable step-size and its application to improve the quality of broadcast-telephone system use in underground coal mines is presented in this paper. The step-size of the proposed algorithm is proportional to not only the norm of the smoothed gradient vector but also the smoothed mean-square error. Simulation results are presented to support the analysis and to compare the performance of the new LMS algorithm with the ordinary LMS, NLMS algorithms. They show that the performance of the new algorithm in stationary as well as in non-stationary environment is superior to ordinary LMS, NLMS algorithms.

Keywords -

Adaptive noise cancellation; Broadcast-telephone system; LMS algorithm; NLMS algorithm
\end{abstract}

\section{Introduction}

The broadcast-telephone system is used for managing, dispatching and ensuring safety in underground coal mines. Mine workers can communicate by two-way audio intercom with their manager through this system. The system is based on Ethernet platform in coal mine and UDP protocol, and converts voice signal to standard IP packet to transmit in Ethernet by using technologies of computer, network, VOIP and embedded system.

However, during the dialogue, the noise generated by the devices in underground coal mine will affect quality of human voice. According to the spectral analysis, the noise includes harmonic components and its spectrum overlaps a spectrum of the human voice and the band of the noise varies with time, so traditional filters as FIR or IIR are not effective. The results of our experiments show that, we can reduce this noise by using an adaptive filter.

One of the most popular algorithms in adaptive signal processing is the least mean square (LMS) algorithm with a constant step-size of Widrow and Hoff [3]. In practical application, a key parameter of LMS algorithm is the step-size. It is well known that, if the step-size is large, the convergence rate of the LMS algorithm will be fast, but the steady-state mean square error (MSE) will be large. On the other hand, if the step-size is small, the steady-state MSE will be small, but the convergence rate will be slow. Thus, the step-size provides a tradeoff between the convergence rate and the steady-state MSE of the LMS algorithm. In recent years, a several variable step-size algorithms based on ordinary LMS algorithm have been proposed [1], [4], [5], [6]. Costa et al. in [1] proposed a noise resilient variable step-size LMS (NRVSS). The NRVSS algorithm is specially indicated for adaptive interference reduction in biomedical application. Ravikanth and Sanket Dessai [2] proposed a design and development of Noise Cancellation System (NCS) to cancel the background noise from the speech signal of speaker in android mobile phones. NCS system has been implemented using an adaptive filter. In [4], the authors proposed an algorithm to remove noise from speech signal in real time environment. The quality of audio signal can be improved by filtering the degraded speech signal through adaptive filters. Fengchun Wang, Jiyan Du [6], proposed the EFLMS (Error Feedback Least Mean Square) algorithm is based on Sigmoid function. In [7], Akash Kashyap, et. al, designed an adaptive filter to remove an unwanted noise which might occur during music recordings, and echo in telephone networks. In [8], Zhenghua Ma; Liujun Yao; Jiongru Zhou; and Dan Kong introduced a design scheme of digital broadcast telephone system of coal mine based on TCP/IP protocol and TMS320DM642 digital processor. The system makes use of audio codec chip TLV320AIC23B to achieve audio codec and adaptive anti-noise algorithm so that the output audio's quality could be good to meet requirements of the voice in the mine, etc.

In this paper, the new algorithm based LMS algorithm where the step-size adjustment is controlled by the mean-square error and the norm of the gradient 
vector is proposed to enhance the convergence rate, steady-state MSE and other performance indexes of the ordinary LMS algorithm in the presence of statistically stationary or non-stationary measurement noise. As will be shown in the simulations, the proposed algorithm has improved performance as compared with existing LMS, NLMS algorithms.

Throughout the paper, the following notations are adopted, Table 1.

Table 1. Notations

\begin{tabular}{ll}
$\|\cdot\|$ & Euclidean norm of a vector \\
$|\cdot|$ & Absolute value \\
$\mathrm{E}[]$. & Expectation operator \\
$\operatorname{Tr}()$. & Trace of a matrix \\
$\mathbf{w}$ & Weight vector $(\mathrm{Lx} 1)$ \\
$\mathbf{p}$ & Cross-correlation vector \\
$\mathbf{R}$ & Auto-correlation matrix (LxL) \\
$\mathrm{e}$ & Scalar-valued error signal \\
$\mathbf{x}$ & Row input vector $(1 \mathrm{xL})$ \\
$(.)^{\mathrm{T}}$ & Transpose of a vector \\
$(.)^{\mathrm{H}}$ & Transpose of a matrix \\
$\mathrm{L}$ & Number taps coefficients \\
$\mathrm{N}$ & Total number of samples \\
$\mathbf{J}$ & Function cost \\
$\mu$ & Step-size paramerter \\
$\nabla$ & Gradient vector \\
$\mathrm{P}_{\mathrm{x}}$ & Average power of the input $\mathbf{x}$ \\
$\delta^{2}$ & Variance \\
$\lambda$ & Eigenvalue \\
\hline
\end{tabular}

\section{The Proposed New LMS Algorithm}

In this section, the novel algorithm is proposed to remove unwanted noise in various environments such as underground coal mines. Figure 1 shows our adaptive noise cancellation for broadcast-telephone system. The adaptive filter consists of two distinct parts: a digital filter with adjustable coefficients $\mathbf{w}$, and an adaptive algorithm which is used to adjust or modify the coefficients of the filter. Two input signals $d_{k}$ (which is received by the microphone $1^{\text {st }}-$ Mic1) and $x_{k}$ (Mic2) are applied simultaneously to the adaptive filter. The signal $d_{k}$ is the contaminated signal containing both the desired signal $s_{k}$ - voice of mine worker - and the noise $\mathrm{n}_{\mathrm{k}}$ - sound of devices in underground coal mine. These two signals $\left(\mathrm{s}_{\mathrm{k}}\right.$ and $\mathrm{x}_{\mathrm{k}}$ ) are assumed to be uncorrelated. The signal $\mathrm{x}_{\mathrm{k}}$ is a measure of the contaminating signal which is correlated in some way with $n_{k}$. The noise $x_{k}$ processed by the digital filter to produce an estimate $n_{k}$ of $n_{k}$. An estimate of the desired signal is then obtained by subtracting the digital filter output $n_{k}$ from $\mathrm{d}_{\mathrm{k}}(1)$.

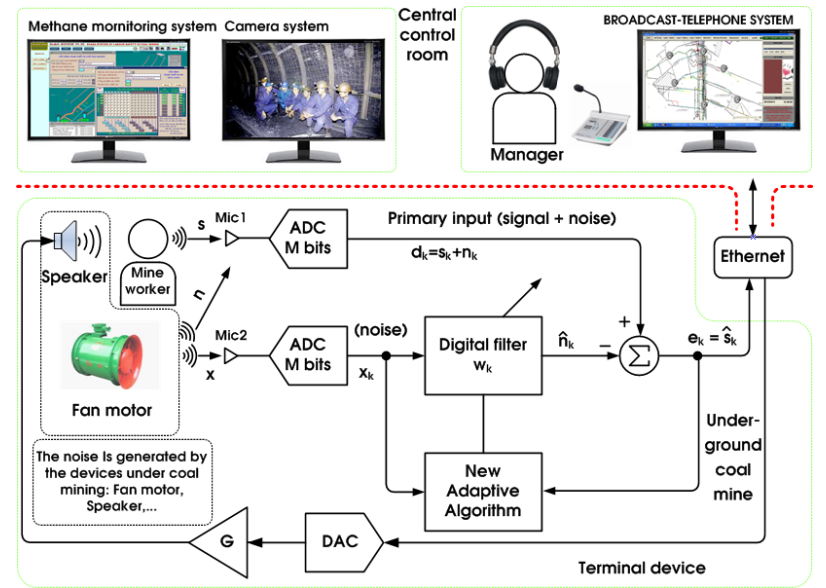

Figure 1. Adaptive noise cancellation for broadcasttelephone system

$$
e_{k}=\hat{s}_{k}=d_{k}-n_{k}=s_{k}+n_{k}-n_{k}
$$

The main objective in noise cancelling is to produce an optimum estimate of the noise in the contaminated signals and hence an optimum estimate of the desired signal is received. This is achieved by using $e_{k}$ in a feedback arrangement to adjust the digital filter coefficients $\mathbf{w}$ (weight vector) via the new adaptive algorithm, to minimize the noise in $\hat{s}[k]$. From (1), we have:

$$
\begin{aligned}
& e_{k}=d-n_{k} \\
& =d_{k}-\mathbf{w}_{k}^{T} \mathbf{x}_{k}=d_{k}-\sum_{i=0}^{L-1} w_{i}[k] x[k-i]
\end{aligned}
$$

The square of the error, we get:

$$
e_{k}^{2}=d_{k}-2 d_{k} \mathbf{x}_{k}^{T} \mathbf{w}_{k}+\mathbf{w}_{k}^{T} \mathbf{x}_{k} \mathbf{x}_{k}^{T} \mathbf{w}_{k}
$$

where $\quad \mathbf{x}_{k}=\left[\begin{array}{lllll}x(k-0) & x(k-1) & \ldots & x(k-L+1)\end{array}\right]^{T} \quad$ is a vector of input signal samples at time $\mathrm{k}$ and $\mathbf{w}_{k}=\left[\begin{array}{lllll}w_{0}(k) & w_{1}(k) & \ldots & w_{-1}(k)\end{array}\right]^{T}$ is a vector containing the coefficients of the digital filter at time $\mathrm{k}$.

The mean square error (MSE), $\mathbf{J}$, is obtained by taking the expectations of both sides of (3).

$$
\begin{aligned}
& \mathbf{J}=E\left[e_{k}^{2}\right]=E\left[d_{k}\right]-2 E\left[d_{k} \mathbf{x}_{k}^{T} \mathbf{w}_{k}\right]+E\left[\mathbf{w}_{k}^{T} \mathbf{x}_{k} \mathbf{x}_{k}^{T} \mathbf{w}_{k}\right] \\
& \mathbf{J}=\delta^{2}-2 \mathbf{w}_{k} E\left[d_{k} \mathbf{x}_{k}^{T}\right]+\mathbf{w}_{k}^{T} E\left[\mathbf{x}_{k} \mathbf{x}_{k}^{T}\right] \mathbf{w}_{k} \\
& \mathbf{J}=\delta^{2}-2 \mathbf{w}_{k} \mathbf{p}_{k}+\mathbf{w}_{k}^{T} \mathbf{R}_{k} \mathbf{w}_{k}
\end{aligned}
$$

From equation (4), we have the gradient of the performance surface:

$$
\begin{aligned}
& \nabla_{k}=\frac{\partial \mathbf{J}}{\partial \mathbf{w}_{k}}=\frac{\delta^{2}}{\partial \mathbf{w}_{k}}-\frac{\partial\left(2 \mathbf{p}_{k}^{T} \mathbf{w}_{k}\right)}{\partial \mathbf{w}_{k}}+\frac{\left(\mathbf{w} \mathbf{R}_{k} \mathbf{w}_{k}\right)}{\partial \mathbf{w}_{k}} \\
& \nabla_{k}=0-2 \mathbf{p}_{k}+2 \mathbf{R}_{k} \mathbf{w}_{k}
\end{aligned}
$$

The weight vector is updated from sample to sample as follows

$$
{ }_{k+1}=\mathbf{w}_{k}-\mu \nabla_{k}
$$

We use instantaneous estimates of cross-correlation 
vector $\mathbf{p}$ and auto-correlation matrix $\mathbf{R}$ by removing the expectation operator in (5). Thus

$$
\begin{aligned}
& \nabla_{k}=-2 \mathbf{p}_{k}+2 \mathbf{R}_{k} \mathbf{w}_{k} \\
& \nabla_{k} \approx-2 \mathbf{x}_{k} d_{k}+\mathbf{x}_{k} \mathbf{x}_{k}^{T} \mathbf{w}_{k} \\
& \nabla_{k} \approx-2 \mathbf{x}_{k}\left(d_{k}-\mathbf{x}_{k}^{T} \mathbf{w}_{k}\right) \\
& \nabla_{k} \approx-2 \mathbf{x}_{k} e_{k}
\end{aligned}
$$

From equations (5), (6) and (7), we have weight vector

$$
\begin{aligned}
& \mathbf{w}_{k+1}=\mathbf{w}_{k}+2 \mu \mathbf{x}_{k} e_{k} \\
& \mathbf{w}_{k+1}=\mathbf{w}_{k}+2 \mu \mathbf{p}_{k}-\mu \mathbf{R}_{k} \mathbf{w}_{k}
\end{aligned}
$$

We consider, $\mathbf{w}_{*}$ is the optimum value of the weight vector. We have weight-error vector at $\mathrm{k}$ and $(\mathrm{k}+1)$ iterations:

$$
\begin{aligned}
& \mathbf{c}=\mathbf{w}_{k}-\mathbf{w}_{*} \\
& \mathbf{c}_{k+1}=\mathbf{w}_{k+1} \quad \mathbf{w}_{*}
\end{aligned}
$$

From equations (8b), (9a), and (9b) we have

$$
\mathbf{c}_{k+1}=\mathbf{c}_{k}-2 \mu \mathbf{R}_{k} \mathbf{c}_{k}-2 \mu \mathbf{R}_{k} \mathbf{w}+2 \mu \mathbf{p}_{k}
$$

Because $\mathbf{w}_{*}$ is the optimum value of the weight vector, from (5) and (10) we get

$$
\begin{aligned}
\mathbf{c}_{k+1} & =\mathbf{c}_{k}-2 \mu \mathbf{R}_{k} \mathbf{c}_{k} \\
\mathbf{c}_{k+1} & =\left(\mathbf{I}-2 \mu \mathbf{R}_{k}\right) \mathbf{c}_{k}
\end{aligned}
$$

The auto-correlation matrix $\mathbf{R}$ may be diagonalized by using a unitary similarity decomposition $\mathbf{R}=\mathbf{Q} \mathbf{\Lambda} \mathbf{Q}^{\mathrm{H}}$, where $\boldsymbol{\Lambda}$ is the diagonal matrix of eigenvalues of $\mathbf{R}$, and the columns of $\mathbf{Q}$ contain the corresponding orthonormal eigenvectors, therefore

$$
\mathbf{c}_{k+1}=\left(\mathbf{I}-2 \mu \mathbf{Q}_{k} \mathbf{\Lambda}_{k} \mathbf{Q}_{k}^{H}\right) \mathbf{c}_{k}
$$

Using the property $\mathbf{Q}^{\mathrm{H}}=\mathbf{Q}^{-1}$, we get

$$
\mathbf{Q}_{k}^{H} \mathbf{c}_{k+1}=\left(\mathbf{I}-2 \mu \boldsymbol{\Lambda}_{k}\right) \mathbf{Q}_{k} \mathbf{c}_{k}
$$

(14)

By defining $\mathbf{v}=\mathbf{Q}^{\mathrm{H}} \mathbf{c}$, we may write

$$
\mathbf{v}_{k+1}=\left(\mathbf{I}-2 \mu \boldsymbol{\Lambda}_{k}\right) \mathbf{v}_{k}
$$

From equation (15) we may write:

$$
\left[\begin{array}{c}
+1 \\
v_{k+1}(1) \\
\ldots \\
v_{k+1}(L-2) \\
v_{k+1}(L-1)
\end{array}\right]=\left[\begin{array}{ccc}
1-2 \mu \lambda^{k} & \ldots & 0 \\
0 & \ldots & 0 \\
0 & \ldots & 1-2 \mu \lambda_{L}^{k}
\end{array}\right]\left[\begin{array}{c}
v_{k}() \\
(1) \\
\ldots \\
v_{k}(L-2) \\
v(L \quad)
\end{array}\right]
$$

where $\lambda_{i}^{k}$ is the $\mathrm{i}^{\text {th }}$ eigenvalue of the correlation matrix $\mathbf{R}_{\mathrm{k}}$.

Equation $\quad(16) \quad \Rightarrow v_{k+1}(i)=\left(1-\mu \lambda_{i}^{k}\right) v_{k}(i) \Rightarrow$ $v_{k+1}(i)=\left(1-2 \mu \lambda_{i}^{k}\right)^{k+1} v_{k}(0)$. Since all eigenvalues of the auto-correlation matrix $\mathbf{R}$ are positive and real, the response $v_{k+1}($ ) will no oscillations. For stability or convergence of the algorithm, the magnitude of the geometric ratio of the above geometric series must be less than 1 for all $\mathrm{i}$.

$$
\begin{gathered}
-1<1-2 \mu \lambda_{i}^{k}<1 \forall i \Rightarrow \mu<\frac{1}{\lambda_{\max }^{k}} \\
\Rightarrow \mu<\frac{1}{\sum_{i=0}^{L-1} \lambda_{i}^{k}}=\frac{1}{\operatorname{tr}\left(\mathbf{R}_{k}\right)}=\frac{1}{L P_{k}}
\end{gathered}
$$

where $P_{x k}=E\left[x^{2}\right]$ is the average power of the input $x_{k}$. Since the trace is just the sum of the $\mathrm{L}$ diagonal elements, $\operatorname{tr}\left(\mathbf{R}_{\mathrm{k}}\right)=\mathrm{LP}_{\mathrm{xk}}$.

Note that:

(1) The norm of the gradient vector will be large initially and converge to a small value, potentially zero, at steady-state;

(2) The polarity of the gradient vector will generally be consistent during the early stage of the adaptive process and change frequently after the system converges;

(3) From equations (4), (6), (17) we have $0 \leq \mathbf{J}\left(\mathbf{w}_{\mathrm{k}+1}\right)<$ $\mathbf{J}\left(\mathbf{w}_{\mathrm{k}}\right)$;

(4) In practice, if the ADC has M-bit data, we have

$$
\begin{aligned}
& \mathbf{J}_{\max } \leq\left(2^{M}\right)^{2} \\
& \Rightarrow \frac{1}{\sum_{j=0}^{N-1} a_{j}} \sum_{j=0}^{N-1} a_{j} e_{k}(j) \quad 2^{2 M} \\
& \Rightarrow \frac{\frac{1}{\sum_{j=0}^{N-1} a_{j}} \sum_{j=0}^{N-1} a_{j} e_{k}^{2}(j)}{2^{2 M+}} \frac{1}{2}
\end{aligned}
$$

(5) If the ADC has M-bit data, from equation (7) so we have

$$
\begin{aligned}
& \| \quad \begin{array}{l}
\| \approx\left|-2 x_{k} e_{k}\right| \quad 2^{2 M+} \\
\sum_{j=0}^{N-1} b_{j} \sum_{j=0}^{N-1} b_{j}\left|x_{k}(j) e_{k}(j)\right| \\
2^{2 M+}
\end{array} \frac{1}{2}
\end{aligned}
$$

Hence, from (17), (20), and (22), we have $\frac{\frac{\sum_{j}^{N-1} b_{j}^{N-} b_{j}\left|x_{k}(j) e_{k}(j)\right|}{2^{2 M+1}}}{L P_{x_{k}}}+\frac{\frac{\frac{1}{N-}_{j=0}^{N}{ }_{j=0}^{1} b_{j} e_{k}^{2}(j)}{2^{2+1}}}{L P_{x_{k}}} \leq \frac{1}{L P_{x_{k}}}$

$$
\begin{aligned}
& \Rightarrow \\
& \frac{\frac{1}{\sum_{j=0}^{N-1} b_{j} \sum_{j=0}^{N-1} b_{j}\left|x_{k}(j) e_{k}(j)\right|-} b_{j} e_{k}^{2}(j)}{2^{2 M+1}}+\frac{2 \frac{1}{2}}{L P_{x_{k}}+1} \leq \frac{1}{L P}
\end{aligned}
$$




$$
\begin{gathered}
\Leftrightarrow \frac{\frac{1}{2^{2 M+} L \sum_{j=0}^{N-1} b_{j}} \sum_{j=0}^{N-1} b_{j}\left|x_{k}(j) e_{k}(j)\right|}{\frac{1}{2^{2 M+} L \sum_{j=0}^{N-1} a_{j}} \sum_{j=0}^{N-1} a_{j} e_{k}^{2}(j)}+ \\
\frac{x_{k}+L^{-1}}{L P_{x_{k}}}
\end{gathered}
$$

Hence, we may be choose step-size by

$$
\mu_{k}=\left\{\begin{array}{l}
\frac{\alpha \sum_{j=0}^{N-1} b_{j}\left|x_{k}(j) e_{k}(j)\right|+\beta \sum_{j=0}^{N-1} a_{j} e_{k}^{2}(j)}{P_{x_{k}}+L^{-1}} \\
\mu_{\max } i f \frac{\alpha \sum_{j=0}^{N-1} b_{j}\left|x_{k}(j) e_{k}(j)\right|+\beta \sum_{j}^{N-1} a_{j} e_{k}^{2}(j)}{x_{k}+L^{-1}} \geq \mu_{\max } \\
\mu_{\min i f} \frac{\alpha \sum_{j=0}^{N-1} b_{j}\left|x_{k}(j) e_{k}(j)\right|+\beta \sum_{j} a_{j} e_{k}^{2}(j)}{x_{k}+L^{-1}} \leq \mu_{\min }
\end{array}\right.
$$

where: $a_{j}$ and $b_{j}$ are coefficients designed as the coefficients of the low-pass filter with cut-off frequency depends on the spectrum of input signal $\mathbf{s}$.

$$
\begin{gathered}
\alpha=\frac{B}{2^{2 M+} L \sum_{j=0}^{N-1} b_{j}} \\
\text { and } \beta=\frac{A}{2^{2 M+} L \sum_{j=0}^{N-1} a_{j}}
\end{gathered}
$$

where: A, and B are gain coefficients. We use the gain coefficients A and B to increase the role of $\mathbf{J}$ as well as the norm of the gradient vector, then we use $\mu_{\max }$ and $\mu_{\min }$ to guarantee bounded.

From the proposed algorithm (26), we have large step-size $\left(\mu_{\max }\right)$ at the early stages of the adaptive process and value of the step-size is decreasing to $\mu_{\min }$ when the system approaches convergence so that we can improve convergence speed and get good value of SNR; In the (26), $a_{j}$ and $b_{j}$ coefficients are designed as the coefficients of the low-pass filter with cut-off frequency depends on the spectrum of $\mathbf{s}$ signal so that the noise of $\mathbf{J}$ and the norm of the gradient vector are reduced.

\section{Simulation Results}

Simulations are performed in this subsection to show the advantages of the proposed algorithm. In simulations, the proposed algorithm (26) will be compared with the LMS, NLMS algorithms.

\subsection{Simulation 1}

In the first simulations, the noise $\mathbf{x}$ is a pseudorandom, zero-mean and unit variance Gaussian process. The noise signal $\mathbf{n}$ is a pseudorandom, zeromean and unit variance Gaussian process. The signal is $\mathrm{s}(\mathrm{n})=1+0.01 * \sin (2 * \mathrm{pi} * 1000 * \mathrm{t}(\mathrm{n}))$. A frequency of $\mathbf{s}$ signal is $1 \mathrm{kHz}$ - in the human voice frequency range, with a length of 1000 samples.

Case 1: In this case, the parameters used in the new algorithm are: $\mathrm{L}=4 ; \mathrm{N}=10 ; \mathrm{a}_{\mathrm{j}}=0.1 ; \mathrm{b}_{\mathrm{j}}=0.1$; $\alpha=5.10^{-3}, \beta=5.10 \quad, \mu_{\max }=0.15 ; \mu_{\min }=8 * 10^{-3}$; The parameter used in the LMS algorithm is $\mu=0.01$; The NLMS algorithm is $\mu_{\text {NLAS }}=\frac{0.01}{\|x\|_{2}^{2}+1}$. The simulation results are presented in Figure 2 and Table 2. Figure 2 shows the MSE obtained from Monte Carlo simulations (100 runs), and the values of SNR are shown in the Table 2.

Table 2. SNR (dB) in case 1

\begin{tabular}{ccccc}
\hline S.No & $\begin{array}{c}\text { Before } \\
\text { filtering }\end{array}$ & $\begin{array}{c}\text { New } \\
\text { algorithm }\end{array}$ & NLMS & LMS \\
\hline 1 & 0.0084 & 21.1687 & 17.0503 & 15.8665 \\
2 & -0.0031 & 21.2747 & 20.7448 & 15.5701 \\
3 & 0.2937 & 19.4356 & 17.1666 & 14.5539 \\
4 & -0.0359 & 14.3001 & 13.3513 & 11.7474 \\
5 & 0.3259 & 19.1568 & 18.3731 & 12.6130 \\
\hline
\end{tabular}

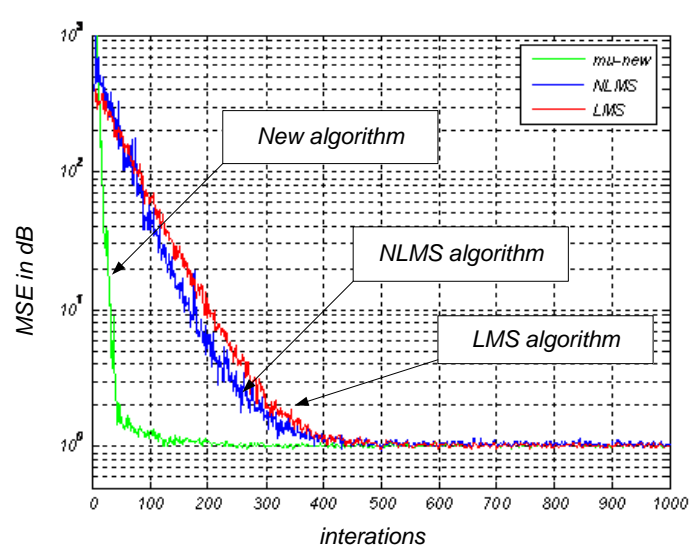

Figure 2. Comparison of MSE and convergence rate of new algorithm and LMS, NLMS in case 1

Case 2: This is the same as the previous case but the parameter used in the LMS algorithm is $\mu=0.08$; The NLMS algorithm is $\mu_{N L M S}=\frac{0.08}{\|x\|_{2}^{2}+1}$. The simulation results are presented in Figure 3 and Table 3. Figure 3 shows the MSE obtained from Monte Carlo 
simulations (100 runs), and the values of SNR are shown in Table 3.

Table 3. SNR $(\mathrm{dB})$ in case 2

\begin{tabular}{ccccc}
\hline S.No & $\begin{array}{c}\text { Before } \\
\text { filtering }\end{array}$ & $\begin{array}{c}\text { New } \\
\text { algorithm }\end{array}$ & NLMS & LMS \\
\hline 1 & -0.1700 & 20.6856 & 9.4027 & 7.1442 \\
2 & 0.4142 & 21.9611 & 9.1564 & 7.1248 \\
3 & -0.1061 & 17.0344 & 8.9119 & 6.5772 \\
4 & 0.1751 & 20.5712 & 9.5833 & 7.2531 \\
5 & -0.3751 & 19.8617 & 8.6202 & 6.3245 \\
\hline
\end{tabular}

According to the simulation results, the performance of the LMS, NLMS methods with parameters as in case 1 has a small MSE and a large SNR, but the convergence rate is very slow. In case 2, we use the large step-size for LMS and NLMS algortihms; therefore the convergence rate increases. Moreover, when we use the large step-size for LMS and NLMS, the SNR value is reduced. The results shows that the new algorithm has better SNR and converges rate than LMS, and NLMS algorithms.

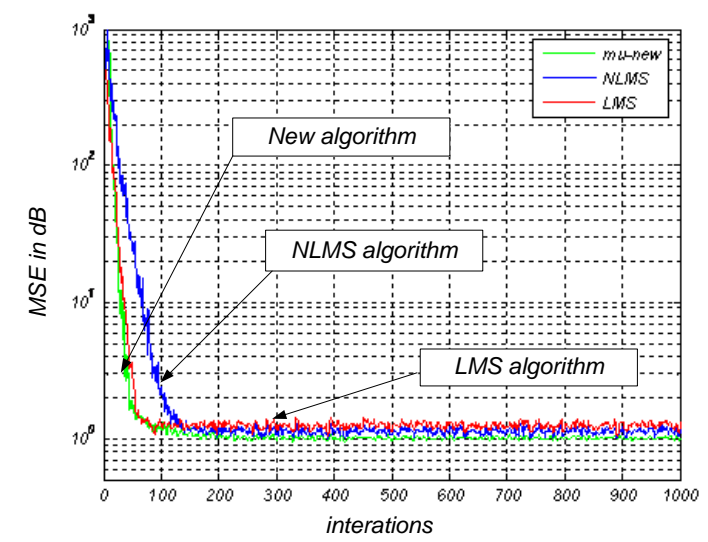

Figure 3. Comparison of MSE and convergence rate of new algorithm and LMS, NLMS in case 2

\subsection{Simulation 2}

In this simulation, the signal and noise are nonstationary random process. The noise $\mathbf{x}$ and $\mathbf{n}$ are a sound of devices in coal mines. They are recorded with a sampling rate of $8,000 \mathrm{~Hz}$, Figure 5. The signal $\mathbf{s}$ is samples of a speech signal which is available from web site www.voiptroubleshooter.com, and the file name is "OSR_us_000_0030_8k.wav", Figure 4. It has a sample rate $\mathrm{Fs}_{\mathrm{s}}=8,000 \mathrm{~Hz}$. Figure 6 shows that the spectrum of the noise overlaps spectrum of the human voice. The parameters used in the new algorithm are: $\mathrm{L}=4 ; \mathrm{N}=$ $10 ; a_{j}=0.1 ; b_{j}=0.1 ; \alpha=5.10^{1}, \beta=2.10^{-} ;$the parameter used in the LMS algorithm is $\mu=0.01$; The NLMS algorithm is $\mu_{N L M S}=\frac{0.08}{\|x\|_{2}^{2}+1}$. In this simulation, we performed experiments with the SNRs (before filtering) are scaled $-14.0990 \mathrm{~dB} ;-8.0784 \mathrm{~dB}$ (Figure 7), -0.1196 $\mathrm{dB} ; 2.8030 \mathrm{~dB} ; 5.9010 \mathrm{~dB}, \ldots$ The simulation results are presented in Figure 8, 9, Figure 10 and Table 4. Figure 8 shows the behavior of the LMS algorithm, the amplitude of the noise is reduced along the horizontal axis. The Output_LMS.wav shows that the noise reduce gradually, and disappeared about 5 seconds. Figure 9 shows the behavior of the NLMS algorithm. In this figure, the amplitude of the noise is reduced quickly than that of Figure 8. However, we still hear some noise when we play Output_NLMS.wav file because the SNR value is not high. It is clear from Figure 10 and Table 4 that the new LMS algorithm performs better than the LMS, NLMS in term of the SNR as well as the convergence rate. It looks like we do not hear the noise when we play the Output_New_Algorith.wav file.

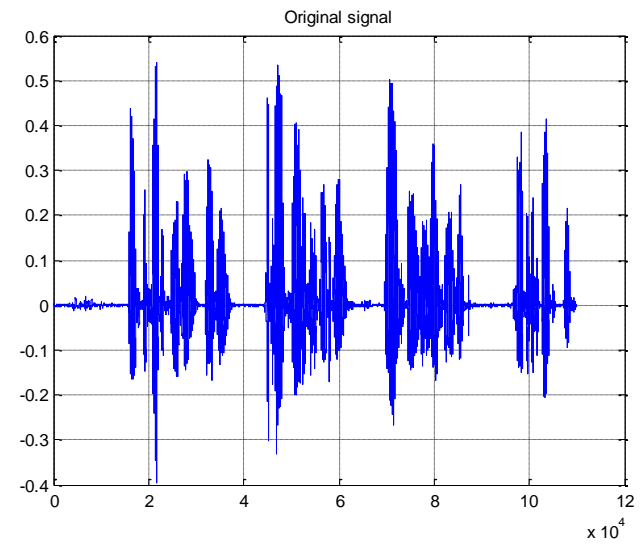

Figure 4. Original signal (OSR_us_000_0030_8k.wav) with 110.000 samples

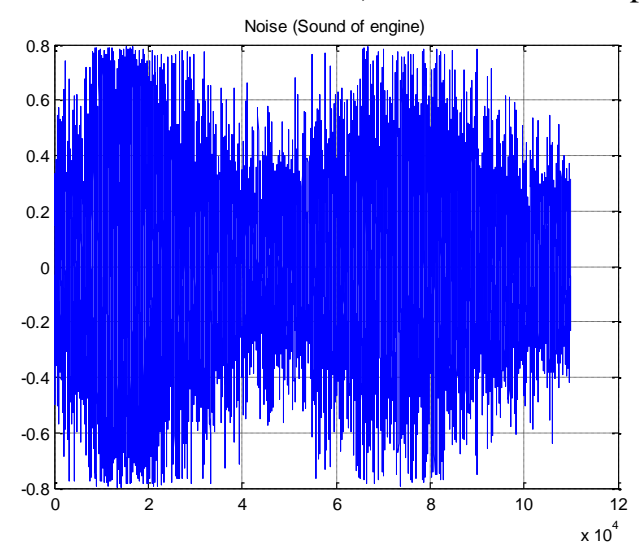

Figure 5. Noise (sound of engine) with 110.000 samples 

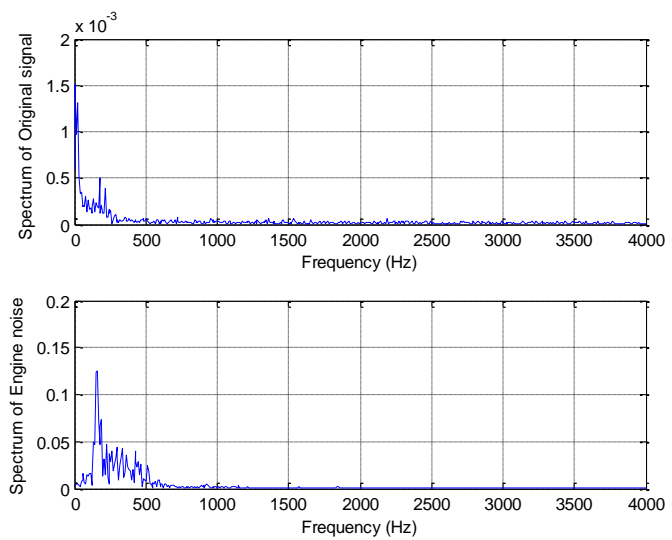

Figure 6. Amplitude spectrums of Original signal and Noise, in the range between 0 and $500 \mathrm{~Hz}$

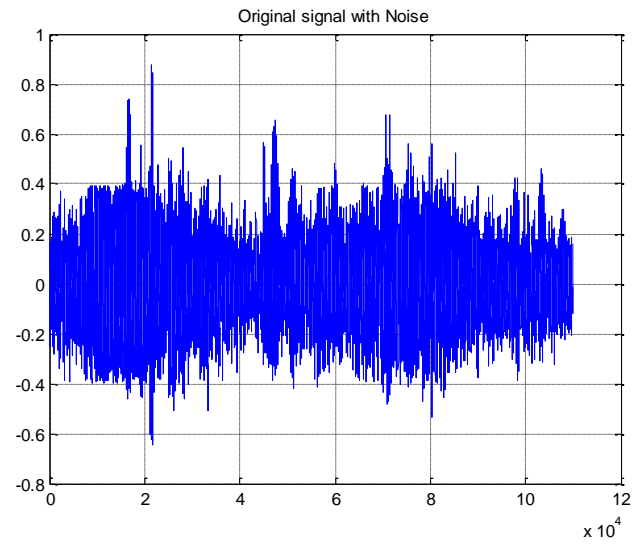

Figure 7. Original signal+noise (sound of engine) with 110.000 samples, $\mathrm{SNR}=-8.0784 \mathrm{~dB}$

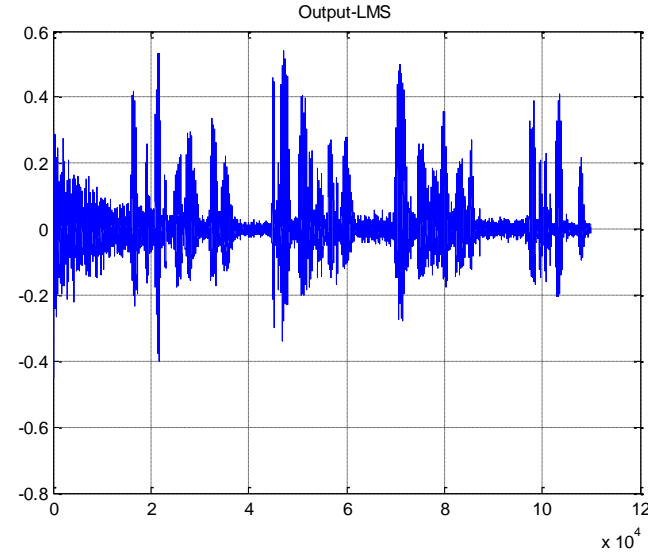

Figure 8. Output signal (Output_LMS.wav) with 110.000 samples, $\mathrm{SNR}=14.8680 \mathrm{~dB}$

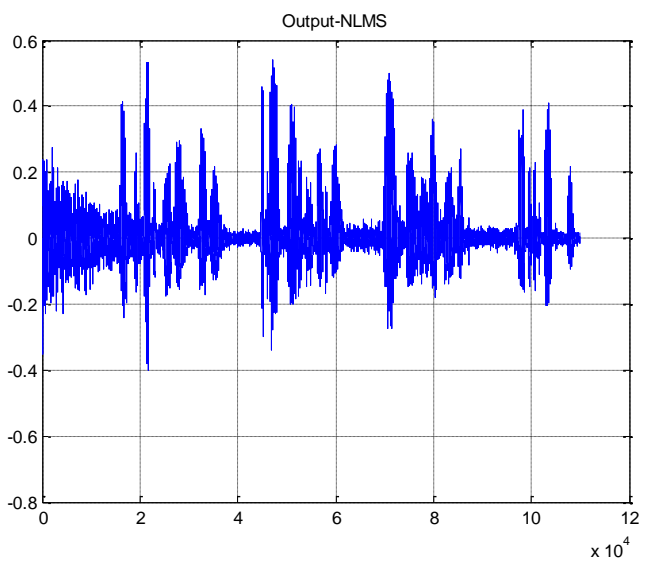

Figure 9. Output signal (Output_NLMS.wav) with 110.000 samples, SNR $=11.9066 \mathrm{~dB}$

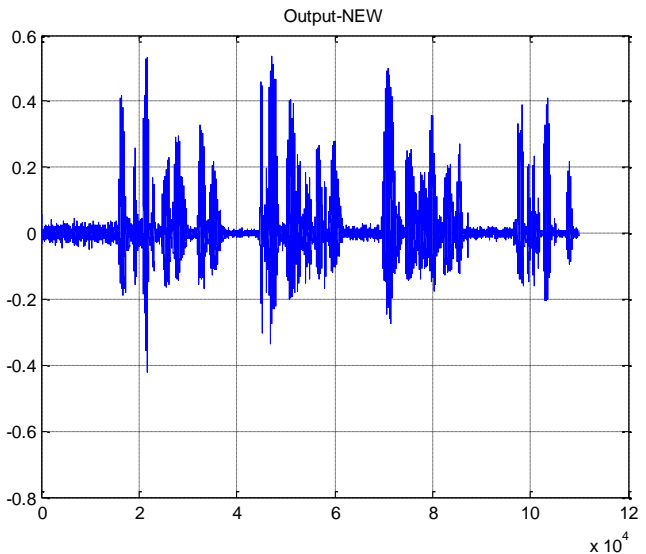

Figure 10. Output signal (Output_New_Algorith.wav) with 110.000 samples, $\mathrm{SNR}=18.6652 \mathrm{~dB}$

Table 4. SNR $(\mathrm{dB})$ in the simulation 2

\begin{tabular}{ccccc}
\hline S.No & $\begin{array}{c}\text { Before } \\
\text { filtering }\end{array}$ & $\begin{array}{c}\text { New } \\
\text { algorithm }\end{array}$ & NLMS & LMS \\
\hline 1 & -14.099 & 15.1303 & 11.5308 & 10.3922 \\
2 & -8.0784 & 18.6652 & 11.9066 & 14.8680 \\
3 & -4.5565 & 19.8141 & 11.9787 & 16.6313 \\
4 & -2.0578 & 20.3001 & 12.0038 & 17.4678 \\
5 & -0.1196 & 20.5445 & 12.0153 & 17.9170 \\
6 & 1.4641 & 20.6831 & 12.0215 & 18.1819 \\
7 & 2.8030 & 20.7687 & 12.0252 & 18.3497 \\
8 & 3.9628 & 20.8252 & 12.0275 & 18.4622 \\
9 & 4.9859 & 20.8643 & 12.0291 & 18.5410 \\
10 & 5.9010 & 20.8924 & 12.0302 & 18.5982 \\
11 & 7.4847 & 20.9292 & 12.0316 & 18.6738 \\
12 & 8.1799 & 20.9417 & 12.0321 & 18.6995 \\
13 & 8.8236 & 20.9516 & 12.0324 & 18.7200 \\
14 & 9.4229 & 20.9595 & 12.0327 & 18.7366 \\
\hline
\end{tabular}

\section{Conclusion}


In this paper the process of noise removal from speech signal to improve a quality of broadcasttelephone system by using the new LMS algorithm is presented. The performance of the new LMS algorithm is better than the LMS, NLMS in term of the SNR and the convergence rate because the step-size (26) is controlled by both the smoothed mean-square error and the norm of the smoothed gradient vector. Simulations show that this algorithm can obtain not only the fast convergence rate but also the large SNR. Thus the theoretical and simulation results prove that the proposed algorithm is the promising method for removing the noise in the signal. Future work will use the new algorithm (26) for echo cancellation to improve audio quality for VoIP-Wifi-Phones use in underground coal mines.

\section{References}

[1] M. H. Costa and C. M. Bermudez, A noise resilient variable step-size LMS algorithm, Signal Processing, vol. 88, pp. 733-748, 2008.

[2] Ravikanth N., Design and development of noise cancellation system for Android mobile phones, Volume 11, Issue 1, Apr 2012.

[3] Widrow, B., and Stearns., S. D. Adaptive signal processing, Englewood Cliffs, NJ: Prentice-Hall, 1985.

[4] Raja and A.Shanmugam, Performance analysis of adaptive algorithms for noise cancellation in speech processing, Journal of Theoretical and Applied Information Technology 20th, February 2013.

[5] S. Zhao and Z. Manb, Variable step-size LMS algorithm with a quotient form, Signal Processing, vol. 89, pp. 67-76, 2009.

[6] Fengchun Wang and Jiyan Du, A variable stepsize LMS adaptive filtering algorithm based on error feedback, International Conference on Education Technology and Information System ICETIS, pp. 812-815, 2013.

[7] Akash Kashyap and Mayank Prasad, Audio noise cancellation using Wiener Filter based LMS algorithm using Labview, International Journal Of Emerging Technology And Advanced Engineering, Volume 3, Issue 3, March 2013.

[8] Zhenghua Ma, Liujun Yao, Jiongru Zhou and Dan Kong, Design of digital broadcast telephone system of coal mine, International Conference on Electronics, Communications and Control ICECC-2011, China, 9-11 Sept. 2011. 